\title{
Basic tests of respiratory function
}

\section{Michael C. F. Pain, Director of Thoracic Medicine, Royal Melbourne Hospital, Melbourne}

\section{SYNOPSIS}

Respiratory function tests are part of the clinical assessment of many respiratory diseases. The tests can measure individual parts of the respiratory process and, therefore, need to be selected appropriately. Spirometry is the basic screening test for assessing mechanical load problems. Arterial blood gas analysis yields considerable information about gas exchange efficiency while tests of gas transfer assess alveolar-capillary surface function. When specifically indicated, assessing bronchial reactivity and the response to exercise can help in the evaluation of breathlessness.

Index words: pulmonary physiology, spirometry, breathlessness.

(Aust Prescr 2000;23:10-2)

\section{Introduction}

In the last 45 years tests of respiratory function have moved from the research laboratories of university departments into the world of everyday practice. This is appropriate as the tests greatly improve diagnostic skills and disease management.

There are many different respiratory function tests, each with strengths and weaknesses. Importantly, a report of respiratory function is only as good as the extent of patient co-operation, the training and skill of the technical staff, the equipment used and the experience of the reporter. Guidelines for the standardisation of testing, equipment performance and normal values have been published and generally accepted. ${ }^{1,2,3}$

Respiratory function tests are an objective measure of abnormal physiology and a means of following a patient's progress (see box). Considerable reliance is placed on respiratory function testing in the assessment of work-related respiratory illness. The tests rarely provide a single clinical diagnosis and must always be interpreted within the full clinical context. They also require co-operation (sometimes maximal

Lung function tests are most useful in the following situations

- the analysis of breathlessness of obscure origin or when the complaint appears out of proportion to the objective clinical evidence

- the quantitation of respiratory impairment (e.g. fitness for surgery, employment or travel)

- following progress or deterioration, either spontaneous or in response to therapy, (e.g. asthma, fibrosing alveolitis, respiratory failure)

- $\quad$ assessment for medicolegal purposes performance) from the patient, and this may limit their usefulness in certain situations.

For the purposes of testing, the respiratory process can be simplified into its major components. Breathing (i.e. ventilation) results when a given respiratory drive interacts with a certain respiratory load. This process requires work and results in gas exchange. Inadequacies in gas exchange should lead to attempts at correction by a readjustment of drive. Direct testing of respiratory drive is not easy. Defects in respiratory drive are usually inferred from monitoring the effect of an abnormal load on ventilation.

\section{Simple measurements of respiratory load}

Increases in the respiratory load to breathing are very common. Resistive load increases, such as asthma, obstructive bronchitis, cystic fibrosis and emphysema, impair airflow. Elastic load increases such as interstitial fibrosis, muscle paralysis and obesity impair lung inflation. The quantitation of respiratory load involves determining the vital capacity and the speed of maximal expiratory flow.

The peak flow meter is widely promoted as a simple lung function monitor. Serial measurements in conditions such as

\section{Fig. 1}

\section{Volume/time curve}

The basic forced expirogram patterns. Usually about $75 \%$ of a normal sized vital capacity is expelled in a second. In airflow obstruction, the amount expelled in one second is a much smaller fraction of the vital capacity. In lung restriction the small vital capacity can be mostly expelled in about a second.

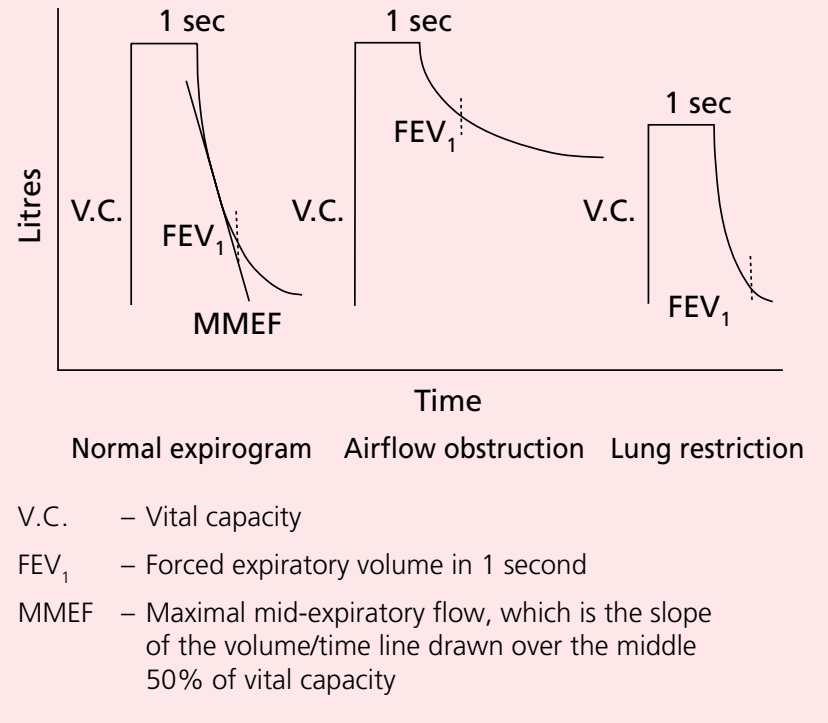


Fig. 2

Maximal expiratory and inspiratory manoeuvres plotted as a flow-volume curve. The dotted vertical line indicates the point of maximal flow after expelling $75 \%$ of the vital capacity.

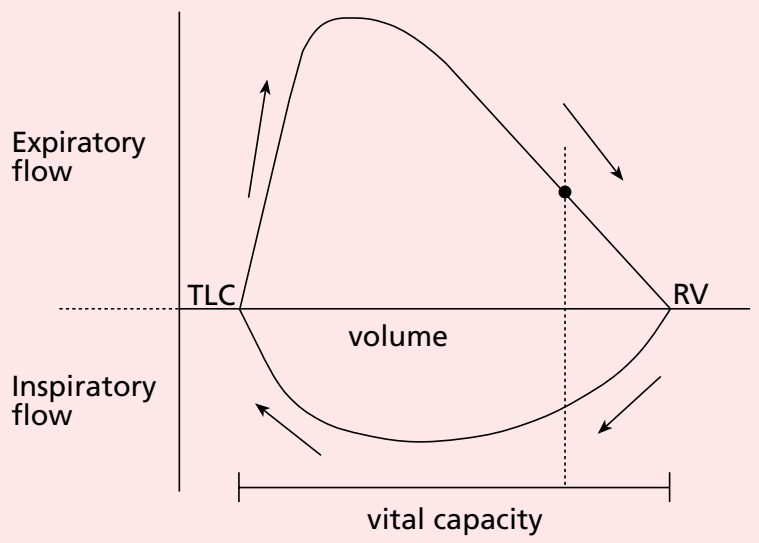

TLC - Total lung capacity

RV - Residual volume

asthma provide valuable information about disease progress. However, peak expiratory flow (the earliest portion of forced expiration) is very effort-dependent. Also, peak flow measurements give no information about elastic load abnormalities.

Load assessment is best done with spirometry. This provides a written record of slow (VC) and/or forced vital capacity (FVC), forced expired volume in one second $\left(\mathrm{FEV}_{1}\right)$ and maximum mid-expiratory flow (MMEF). Most modern electronic spirometers plot volume against flow rate and permit inspiratory as well as expiratory manoeuvres. The machine makes the calculations and corrections and prints out the measured indices.

Expiratory spirometric patterns (Figs. 1 and 2) can be classified as:

Normal Vital capacity within the statistical normal range for height, age and gender. A normal proportion of the $\mathrm{VC}$ can be forcibly expelled within a second (i.e. $\mathrm{FEV}_{1} / \mathrm{VC}$ is $70-80 \%$ ).

Obstructive $\mathrm{FEV}_{1} / \mathrm{VC}$ is below normal. Vital capacity may also be below normal.

Restrictive Vital capacity below the statistical normal range. $\mathrm{FEV}_{1} / \mathrm{VC}$ is higher than normal and may be $100 \%$. $\begin{array}{ll}\text { Mixed } & \text { Vital capacity below normal and } \mathrm{FEV}_{1} / \mathrm{VC} \\ \text { obstructive } & \text { below } 70 \% .\end{array}$

and restrictive

In severe obstruction, the vital capacity may be very reduced. Deciding if a restrictive or obstructive process predominates, may need the measurement of static lung volumes (functional residual capacity or residual volume) to assess the degree of hyperinflation. In practice, this distinction can usually be made clinically and static lung volume measurement is only occasionally important.
Diseases predominantly involving the very small airways (bronchiolitis, interval asthma) produce recognisably distinctive spirometric abnormalities. Indices of small airway function include the MMEF and the expiratory flow during the last phase of expiration. On an expiratory flow-volume curve, small airway abnormality is shown as a concavity in the descending limb and a reduction in the measured flow at some point in the expired vital capacity - often the flow at $75 \%$ vital capacity. These simple tests of small airway function are only useful if there is not concomitant larger airway obstruction.

Forced inspiratory volume measurements, when contrasted with equivalent expiratory volumes, can detect the presence of upper, extrathoracic airway obstruction such as retrosternal goitre or vocal cord paralysis. This form of obstruction is less during expiratory manoeuvres so that the ratios of $\mathrm{FIV}_{1} / \mathrm{FEV}_{1}$, MMIF/MMEF and flows at $75 \%$ vital capacity on an inspiratory and expiratory flow-volume curve become less than unity.

\section{Asthma}

Asthma is a common cause of airflow limitation. The reversibility of the airways obstruction is usually assessed by spirometry before and after a bronchodilator aerosol. An increase of $10 \%$ or more in either vital capacity or $\mathrm{FEV}_{1}$ is taken to indicate significant reversibility, although, of course, not necessarily the maximum reversibility achievable.

When the suspicion of asthma is not confirmed by spirometry, a challenge procedure can be used to assess abnormal bronchial reactivity. This may involve the patient exercising or inhaling histamine, methacholine, hypertonic saline or cold air. Each challenge has its own protocol and risks, and these challenges are best performed in a well-supervised laboratory. Bronchial hyperreactivity is not synonymous with asthma. Although the vast majority of patients with ongoing asthma will have brisk reactivity, most people with a past history of asthma will have intermediate reactivity and some asymptomatic people with no past history will have a degree of bronchial reactivity. Bronchial reactivity is often expressed as the percentage concentration or dose of an agent that produces an acute fall of $20 \%$ in $\mathrm{FEV}_{1}\left(\mathrm{PC}_{20}\right.$ or $\left.\mathrm{PD}_{20}\right)$. Laboratories performing these challenges will usually have established their 'normal reactivity' values.

\section{Simple measurements of gas exchange}

Normal gas exchange requires adequate alveolar ventilation, normal ventilation/blood flow relationships and adequate alveolar-capillary membrane surface area. There are tests of varying sophistication which specifically examine each of these functions.

\section{Alveolar ventilation}

This is not easy to measure directly, as it is not a simple function of the volume of expired air passing the mouth each minute (i.e. the minute ventilation). The size of the dead space (alveolar dead space, connecting tubing volume and tracheobronchial tree) is often uncertain. This uncertainty, combined with the influence of the breathing pattern, means that minute ventilation may be a very misleading estimate of 
alveolar ventilation. To overcome this difficulty, the arterial carbon dioxide tension is used as an inversely proportional index of 'effective' alveolar ventilation. Hence, a normal arterial carbon dioxide tension is taken to indicate satisfactory alveolar ventilation. Elevated or reduced carbon dioxide tensions reflect alveolar hypoventilation or hyperventilation respectively.

\section{Ventilation/blood flow relationships}

These are most simply assessed by considering the lungs as a gas exchanger. Its efficiency is rated by the size of the difference between the amounts of oxygen and carbon dioxide in the blood and in the air. If the lungs are working efficiently the differences in composition will be small. Non-uniformity of ventilation/blood flow ratios will result in abnormally wide differences - the alveolar-arterial $\mathrm{PO}_{2}$ and arterial-alveolar $\mathrm{PCO}_{2}$ gradients will be abnormal. The oxygen tension gradient is normally less than $10 \%$ of the inspired oxygen tension. This simple index can be calculated using the alveolar gas equation (see box).

\section{Alveolar-capillary surface area}

This is assessed by one of several techniques measuring the uptake of carbon monoxide, a gas with affinity for blood and which is easily analysed. Although sometimes designated as tests of diffusion, these techniques are much more influenced by effective alveolar-capillary area and therefore are now more commonly termed gas transfer tests. Although many factors influence the result, these tests are usually abnormal in diffuse interstitial inflammatory and fibrotic processes and in emphysema. They are useful in the subclassification of restrictive conditions (those with and without gas transfer impairment) and in determining the probable extent of emphysema in patients with chronic airflow obstruction. They are commonly used in following patients' response to therapy in such conditions as sarcoidosis and fibrosing alveolitis.

\section{Example: simplified calculation of the alveolar-arterial oxygen tension gradient}

Given data: arterial oxygen tension $=55 \mathrm{mmHg}$ arterial carbon dioxide tension $\left(\mathrm{P}_{\mathrm{a}} \mathrm{CO}_{2}\right)=55 \mathrm{mmHg}$ inspired oxygen concentration $=21 \%$ (room air)

hence inspired oxygen tension $\left(\mathrm{P}_{1} \mathrm{O}_{2}\right)=150 \mathrm{mmHg}$ $\left(21 / 100\right.$ of (barometric pressure $-47^{*}$ ) or multiply concentration by 7.1 if at sea level)

Derive alveolar oxygen tension $\left(\mathrm{P}_{\mathrm{A}} \mathrm{O}_{2}\right)$ from the alveolar gas equation

$$
\mathrm{P}_{\mathrm{A}} \mathrm{O}_{2}=\mathrm{P}_{1} \mathrm{O}_{2}-\mathrm{P}_{\mathrm{a}} \mathrm{CO}_{2} / \mathrm{R}
$$

( $R$ is the gas exchange ratio and is assumed to be 0.8 )

$$
\begin{aligned}
& =150-55 \times 1.25 \\
& =81 \mathrm{mmHg}
\end{aligned}
$$

Hence the alveolar-arterial PO2 gradient is $81-55=\mathbf{2 6} \mathbf{~} \mathbf{m m g}$

Interpretation: As well as ventilatory failure, as demonstrated by the alveolar hypoventilation, there is also a degree of ventilation/ blood flow mismatching since the gradient is greater than normal $(<15 \mathrm{mmHg}$ ).

* $47 \mathrm{mmHg}$ is the water vapour pressure at $37^{\circ} \mathrm{C}$

\section{Simple exercise testing}

Tests performed during exercise provide information about overall fitness and the appropriateness of cardiorespiratory responses. They can be elaborate procedures following cardiac output, pulmonary haemodynamics, gas exchange and anaerobic metabolism measurements at varying grades of exercise, but this type of study has little place in everyday practice. Observations made during a six-minute walk test can provide useful objective information provided the subject is induced to co-operate fully. The actual distance walked, the degree of breathlessness experienced and the change in blood oxygen level (assessed by portable oximetry) are data which can be obtained simply. These data are required before some authorities will agree to provide portable domiciliary oxygen. The extent of exercise limitation due to mechanical load excess agrees reasonably well with the degree of impairment on spirometry. ${ }^{4}$

\section{When to use respiratory function tests}

The most common reason for studying pulmonary function is in the analysis of breathlessness. The application of simple tests of load (spirometry ${ }^{3}$ ), gas exchange (arterial blood gas analysis ${ }^{5}$ ) and gas transfer will usually allow conclusions as to whether or not the complaint is reasonably based.

In hospital practice, the gas exchanging aspects of pulmonary function become important in the assessment and management of acute respiratory failure.

Respiratory function tests are also widely used to assess fitness for surgery, fitness to undertake certain occupations or to assess the degree of impairment in work-related lung conditions.

\section{REFERENCES}

1. American Thoracic Society. Standardization of Spirometry. 1994 Update. Am J Respir Crit Care Med 1995;152:1107-36.

2. American Thoracic Society. Single-breath carbon monoxide diffusing capacity (transfer factor). Recommendations for a standard technique. 1995 Update. Am J Respir Crit Care Med 1995;152:2185-98.

3. Pierce R, Johns DP. Spirometry: the measurement and interpretation of ventilatory function in clinical practice. Melbourne: National Asthma Campaign; 1995.

4. McGavin CR, Artvinli M, Naoe H, McHardy GJ. Dyspnoea, disability, and distance walked: comparison of estimates of exercise performance in respiratory disease. Br Med J 1978;2:241-3.

5. Rubinfeld AR. Blood gas measurements: an aid to their clinical application. Med J Aust 1975;2:309-13.

FURTHER READING

Bates DV. Respiratory function in disease. 3rd ed. Philadelphia: WB Saunders; 1989.

\section{Self-test questions}

The following statements are either true or false (answers on page 23)

3. Healthy adults usually cannot forcibly expel more than $60 \%$ of the vital capacity within one second $\left(\mathrm{FEV}_{1}\right)$.

4. To be meaningful, spirometry demands total co-operation from the patient. 